\title{
Effect of Plasticity on Residual Stresses Obtained by the Incremental Hole-drilling Method with 3D FEM Modelling
}

\author{
Evy Van Puymbroeck ${ }^{1, a^{*}}$, Wim Nagy ${ }^{1, \mathrm{~b}}$ and Hans De Backer ${ }^{1, \mathrm{c}}$ \\ ${ }^{1}$ Ghent University, Department of Civil Engineering EA15, Technologiepark 904, 9052 Ghent, \\ Belgium \\ aEvy.VanPuymbroeck@UGent.be, 'Wim.Nagy@UGent.be, 'Hans.DeBacker@UGent.be
}

Keywords: Residual Welding Stresses, Incremental Hole-drilling Method, FEM, Plasticity Effects

\begin{abstract}
The incremental hole-drilling method is used to determine high residual welding stresses in an orthotropic bridge deck. When comparing the measurement results with a theoretical residual stress distribution of an orthotropic steel deck, a large difference in sign and magnitude of the residual stress values is observed. These measurement results are presented in another paper [1]. The test method, specified in ASTM E837-13a only applies when the material behavior is linear-elastic. Relaxed-plastic strain can be detected in the region of the bored hole for the evaluation of high residual welding stresses. This plastic behavior can result in a significant error of the residual stresses. In this paper, the hole-drilling procedure is simulated and the effect of plasticity on the determination of residual stresses is studied with three-dimensional finite element modelling. The calculation software Siemens NX 9.0 is used to simulate the hole-drilling procedure with both linearelastic and elastic-plastic material behavior. First, a 3D model is set up for uniform in-depth residual stress fields with a linear-elastic material behavior to determine the calibration coefficients. The same model is used to determine similar calibration coefficients but this time with a simplistic model of material plasticity. The effect of plasticity on the uniform in-depth residual stresses is determined. The residual stresses obtained under the assumption that the material behavior is linear-elastic are an overestimation. In future research, residual strains for non-uniform in-depth residual stresses can also be studied with similar models. This will result in a more accurate determination of the residual weld stresses present in bridge constructions.
\end{abstract}

\section{Introduction}

The incremental hole-drilling method is widely used for measuring residual stresses in steel components. Plastic relaxed strain can be induced in the region of the borehole. Since this test method only applies when the material behavior remains linear-elastic, significant errors can be introduced by this plastic behavior during the determination of residual stresses [2]. In this paper, the effect of plasticity on the determination of residual stresses for uniform in-depth residual stresses is studied.

\section{Incremental hole-drilling method}

The residual stresses introduced by a welding operation can be evaluated making use of the incremental hole-drilling technique. A small hole is drilled through the center of a strain gauge rosette into the test material (Fig. 1) [3]. The surface material of the test specimen has to be exposed by drilling only through the material of the strain gauge rosette. This drilling depth is called zero depth. The initial uncertainty of the separation of the cutter from the outer surface by the strain gauge rosette and coating can be disregarded by establishing this zero depth [4].

The relieved surface strains caused by the introduction of a hole in a series of small steps are recorded. Residual stresses are calculated according ASTM E837-13a [3] taking into account the measured strains and calibration coefficients. Reliable measurements are only achieved by limiting 
the residual stresses to $80 \%$ of the material's yield stress in order to take into account that the test method only applies when material behavior is linear-elastic [3]. However, the level of residual stress can be comparable with the material yield stress and then, the stress concentration due to the introduction of the hole produces zones in which the elastic limit of the material is reached. The plastic region arises at the lower circumference of the hole and when the hole depth is increased, it spreads towards the strain gauges located on the strain gauge rosette.

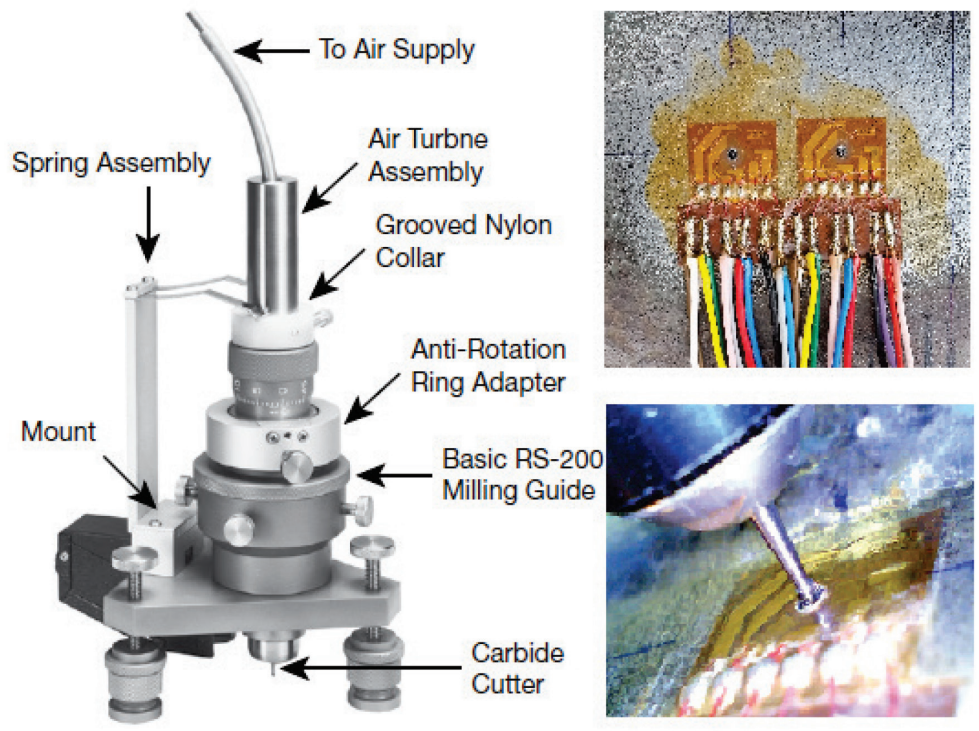

Fig. 1. Hole-drilling machine (left) and hole-drilled strain gauge rosette (right)

A type A strain gauge rosette which follows the Rendler and Vigness [5] geometry is considered in this paper to determine the effect of plasticity on the determination of residual stresses with the incremental hole-drilling method. This pattern is available in several different sizes and is recommended for general-purpose use [3]. The strain gauge rosette type CEA-06-062UL-120 is used for the finite element modelling. A schematic representation of the geometry of a strain gauge rosette used for hole drilling is shown in Fig. 2(a) and a detail of one strain gauge is shown in Fig. 2(b). The dimensions of a strain gauge for the considered strain gauge rosette can be found in Table 1 . The hole diameter of this strain gauge rosette is $2 \mathrm{~mm}$ while the maximum hole-drilling depth is $1 \mathrm{~mm}$.
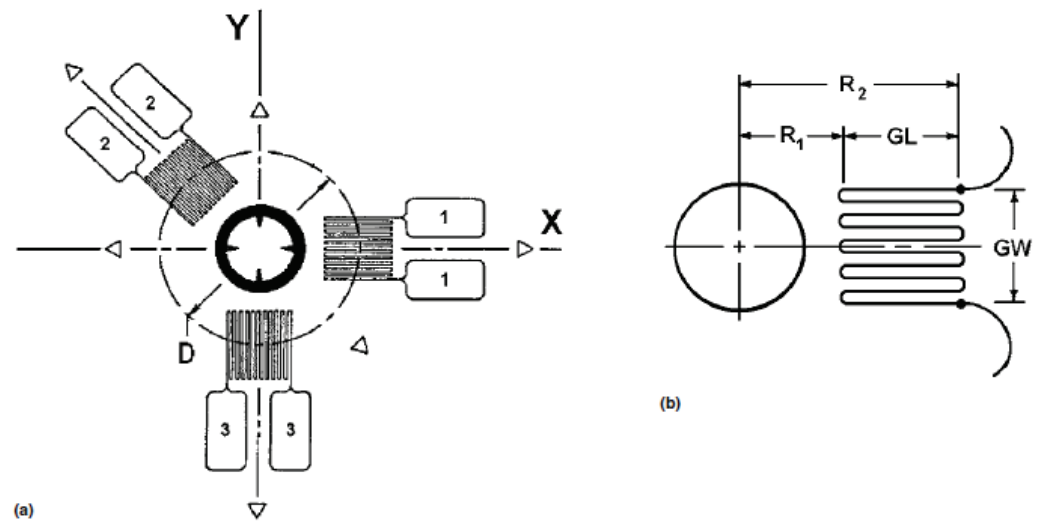

(b)

Fig. 2. Schematic geometry of strain gauge rosette (a) and detail of one strain gauge (b) [2] 
Table 1. Strain gauge rosette dimensions in $\mathrm{mm}$

\begin{tabular}{|l|l|l|l|l|l|}
\hline Rosette Type & $\mathrm{D}$ & $\mathrm{GL}$ & $\mathrm{GW}$ & $\mathrm{R}_{1}$ & $\mathrm{R}_{2}$ \\
\hline CEA-06-062UL-120 & 5.13 & 1.59 & 1.59 & 1.77 & 3.36 \\
\hline
\end{tabular}

\section{D finite element model}

A three dimensional finite element model is set up in Siemens NX 9.0 to evaluate the plastic relaxed strain readings of the considered strain gauge rosette. The geometry of the strain gauge rosette is exactly modelled by defining element nodes corresponding with the number and length of grid lines and their spacing. The strain gauge rosette's material and its bonding to the test surface is not taken into account. It is assumed that the measured strains by the strain gauge rosette are identical to the relaxed strains of the test specimen [6]. Nodal displacements for both ends of every grid line are simulated and they are used to calculate the radial strains.

The hole-drilling procedure is simulated by deactivating elements with the element birth/death feature of the Nastran Solver in NX. Element layers are incrementally deactivated in a number of steps corresponding to the hole-depth increment specified in ASTM E837-13a. For each step, strains are simulated which are used to calculate residual stresses according to the ASTM standard. Only radial strains 1 and 3 (Fig. 2 (a)) will be considered for the evaluation of the residual stresses since the only interest are the normal $\mathrm{x}$ - and y-stresses. The used mesh for the model is shown in Fig. 3 and it contains 436410 three-dimensional elements.

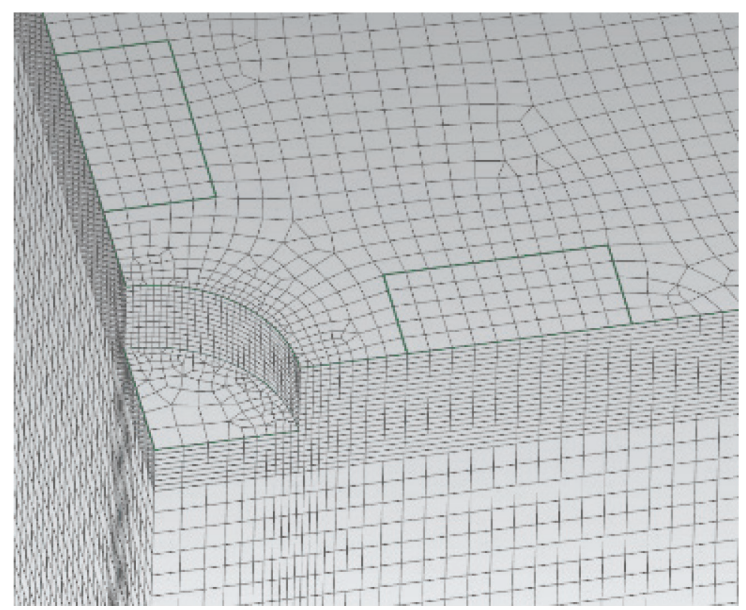

Fig. 3. Mesh of finite element model with drilled hole

Geometry, constraints, material properties and loading conditions. The hole-drilling simulation of a flat plate with a thickness of $6 \mathrm{~mm}$ is performed. The effect of increasing the plate thickness is studied and it has no influence (less than 1\%) on the results. Due to symmetry, only a quarter of the total plate is modelled. Therefore, symmetry constraints are applied on the sides of the model where the hole will be situated which means that the displacements along $\mathrm{x}$ - and $\mathrm{y}$-axes are zero. The displacement of the bottom surface along the z-direction is set to zero. The model width is chosen 15 times the hole diameter and the thickness is larger than the rosette's mean diameter according to the minimum thickness for thick plates recommended by ASTM E837-13a. The steel grade S235 is used as material for the modelling. The presence of a residual stress field is simulated by imposing a uniform pressure distribution of 50MPa on the sides not containing the drilled hole.

In order to make the distinction between linear-elastic and elastic-plastic material behavior, a different stress-strain curve is specified. For linear-elastic material behavior, a linear stress-strain curve is specified until the yield stress is reached (Fig. 4). Elastic-plastic material behavior is 
specified with bilinear stress-strain curve (Fig. 5) and isotropic strain hardening plasticity model. A simplistic model of material plasticity has been used.

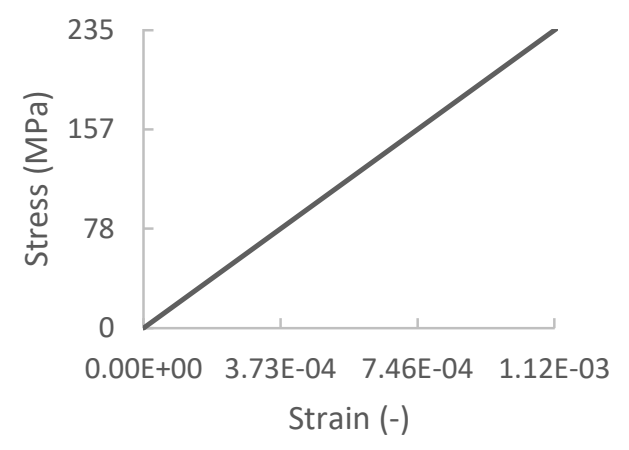

Fig. 4. Linear-elastic stress-strain behavior

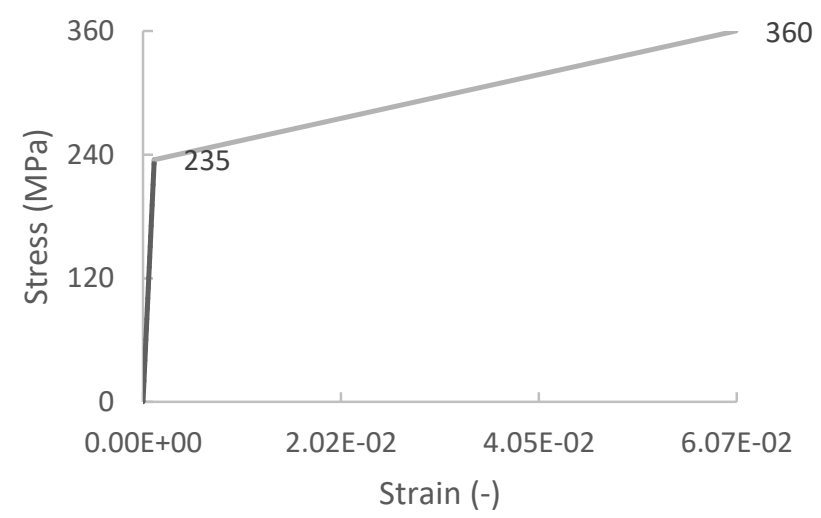

Fig. 5. Elastic-plastic stress-strain behavior

Residual stress calculation. The principal residual stresses $\sigma_{x}$ and $\sigma_{y}$ can be calculated from the measured relaxed strains $\epsilon_{1}$ and $\epsilon_{3}$, the calibration coefficients $a$ and $b$, the material's Young's modulus $E$ and Poisson's ratio $v$. The following relationships apply:

$$
\sigma_{x}=-\frac{E\left(\epsilon_{1}+\epsilon_{3}\right)}{2 a(1+v)}-\frac{E\left(\epsilon_{1}-\epsilon_{3}\right)}{2 b} \quad \sigma_{y}=-\frac{E\left(\epsilon_{1}+\epsilon_{3}\right)}{2 a(1+v)}+\frac{E\left(\epsilon_{1}-\epsilon_{3}\right)}{2 b}
$$

The calibration coefficients are calculated with finite element modelling by applying a known uniform stress distribution on the sides not containing the hole and evaluating the relaxed strains [2]. These imposed pressures are assumed to be equal to the principal residual stresses before a hole was drilled [7].

\section{Determination of calibration coefficients}

Linear-elastic material properties. First, a test piece with uniform in-depth residual stress field is considered in order to numerically calibrate the hole-drilling process and determine the calibration coefficients. A linear-elastic material law is used and the uniform residual stress field is split up into hydrostatic $\left(\sigma_{x}=\sigma_{y}\right)$ and deviatoric $\left(\sigma_{x}=-\sigma_{y}\right)$ residual stress fields. The model with hydrostatic residual stress field is used to determine the calibration coefficient $a$ and the model with deviatoric residual stress field results in calibration coefficient $b$. Both models are evaluated for ten depth-step increments of $0.1 \mathrm{~mm}$ until a final hole depth of $1 \mathrm{~mm}$ is reached. The relaxed strains for each depthstep are evaluated and the calibration coefficients are determined. In Table 2 the calibration coefficients determined with the 3D-model are shown. The results for elastic-plastic material behavior are also already mentioned.

When a comparison is made between the calibration coefficients obtained by the 3D model with linear-elastic material behavior and the coefficients specified in ASTM, the results should be the same. However, the calculated coefficients from the 3D model with linear-elastic material behavior are on average $4 \%$ smaller than the theoretical ones tabulated in ASTM. The difference becomes larger when the hole depth increases and is caused by the location of the strain gauge rosette on the surface. The relaxed strain at deeper hole depth increments has less contribution to the strain response since the distance to the strain gauge rosette increases when reaching deeper hole depths. The determination of the calibration coefficients is dependent on geometric variables including plate thickness, hole diameter and drilling depth. Errors in strain measurement, hole depth and geometry cause also a difference between calculated and theoretical calibration coefficients. The level of uniform residual stress applied in the model also influences the calibration coefficients. Aoh and Wei 
[8] have shown that calibration coefficient $a$ becomes ill conditioned faster than calibration coefficient $b$ which can also can be noticed in Table 2 .

When a comparison is made with calibration coefficients obtained from other studies [8], similar trends and error rates were observed in the experimental calibration by different research works. It is therefore concluded that the coefficients calculated with the specified 3D FEM model are in agreement with the conventional coefficients of the incremental hole-drilling method. Therefore, this model will be used to determine the effect of plasticity on residual stresses obtained by the incremental hole-drilling method.

Table 2. Calibration coefficients

\begin{tabular}{|c|c|c|c|c|c|c|}
\hline & \multicolumn{2}{|c|}{$\begin{array}{c}\text { ASTM E837-13a (linear- } \\
\text { elastic material behavior) }\end{array}$} & $\begin{array}{c}\text { NX9 Linear-elastic material } \\
\text { behavior }\end{array}$ & \multicolumn{2}{c|}{$\begin{array}{c}\text { NX9 Elastic-plastic material } \\
\text { behavior }\end{array}$} \\
\hline $\begin{array}{c}\text { Hole } \\
\text { depth } \\
{[\mathrm{mm}]}\end{array}$ & $\begin{array}{c}\text { Calibration } \\
\text { coefficient } a\end{array}$ & $\begin{array}{c}\text { Calibration } \\
\text { coefficient } b\end{array}$ & $\begin{array}{c}\text { Calibration } \\
\text { coefficient } a\end{array}$ & $\begin{array}{c}\text { Calibration } \\
\text { coefficient } b\end{array}$ & $\begin{array}{c}\text { Calibration } \\
\text { coefficient } a\end{array}$ & $\begin{array}{c}\text { Calibration } \\
\text { coefficient } b\end{array}$ \\
\hline 0.1 & 0.015 & 0.028 & 0.016 & 0.028 & 0.016 & 0.027 \\
\hline 0.2 & 0.036 & 0.066 & 0.037 & 0.065 & 0.036 & 0.062 \\
\hline 0.3 & 0.059 & 0.110 & 0.058 & 0.108 & 0.056 & 0.102 \\
\hline 0.4 & 0.083 & 0.155 & 0.079 & 0.153 & 0.076 & 0.144 \\
\hline 0.5 & 0.104 & 0.200 & 0.097 & 0.197 & 0.094 & 0.186 \\
\hline 0.6 & 0.124 & 0.242 & 0.113 & 0.238 & 0.109 & 0.225 \\
\hline 0.7 & 0.140 & 0.280 & 0.125 & 0.276 & 0.122 & 0.260 \\
\hline 0.8 & 0.154 & 0.314 & 0.135 & 0.309 & 0.131 & 0.292 \\
\hline 0.9 & 0.165 & 0.343 & 0.143 & 0.338 & 0.139 & 0.319 \\
\hline 1 & 0.173 & 0.370 & 0.148 & 0.363 & 0.144 & 0.341 \\
\hline
\end{tabular}

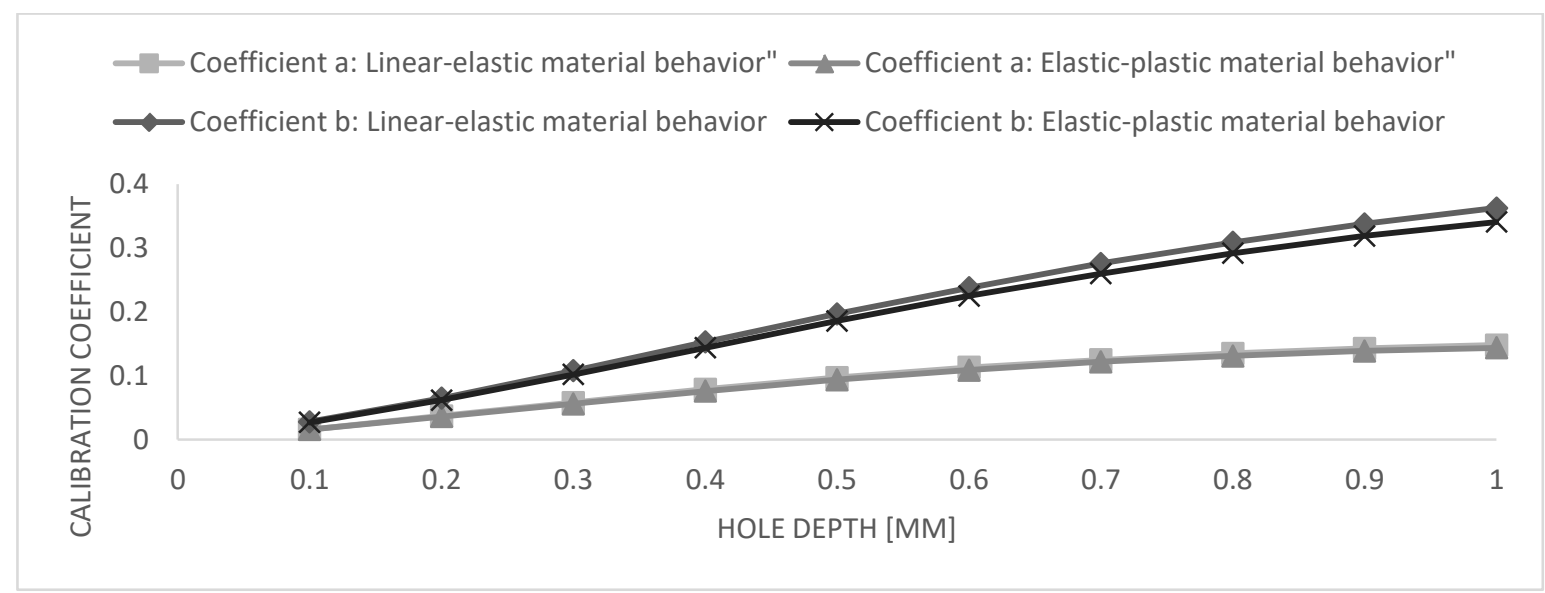

Fig. 6. Comparison between linear-elastic and elastic-plastic material behavior

Elastic-plastic material properties. A comparison is made between the calibration coefficients obtained with linear-elastic and elastic-plastic material properties and the results are shown in Fig. 6. It shows that calibration coefficients are both smaller for the elastic-plastic material behavior. The difference between linear-elastic and elastic-plastic values also increases when the hole depth is increased. A smaller calibration coefficient means that the residual strains and their corresponding residual stresses are lower than the theoretical ones. Taking the effect of plasticity into account, the residual stresses are lower than expected. This means that the residual stresses obtained under the 
assumption that the material behavior is linear-elastic are an overestimation. For calibration coefficient $a$, the mean error rate between linear-elastic and elastic-plastic material behavior is $2.74 \%$ while the mean error rate for calibration coefficient $b$ is equal to $5.37 \%$. The effect of plasticity is larger for deviatoric residual stress fields.

\section{Conclusion}

The hole-drilling procedure is simulated for a uniform in-depth residual stress field and the effect of plasticity is determined based on calibration coefficients. Lower residual stresses are present when elastic-plastic material behavior is assumed. The plasticity introduces relaxation of the material and by ignoring this, the residual stresses will be overestimated by specifying linear-elastic material behavior.

\section{References}

[1] Van Puymbroeck, E., Nagy, W. and De Backer, H., Determination of residual stresses with the incremental hole-drilling method to improbe the design of critical components in bridge construction, 16th International Conference and Exhibition : Structural Faults \& Repair, 2016.

[2] Beghini, M., Bertini, L. and Santus, C., A procedure for evaluating high residual stresses using the blind hole drilling method including the effect of plasticity, The Journal of Strain Analysis for Engineering Design, Vol. 45(4), 2010. http://dx.doi.org/10.1243/03093247JSA579

[3] ASTM E837-13a, Standard Test Method for Determining Residual Stresses by the Hole-Drilling Strain-Gage Method, ASTM International, 2015.

[4] Vishay Measurements Group, Model RS-200 Milling Guide Instruction Manual Version 2.0, Micro-Measurements, USA, 2011.

[5] Rendler, N.J. and Vigness, I., Hole-drilling strain-gage method of measuring residual stresses, Experimental Mechanics, Volume 6, Issue 12, pp 577-586, 1966. http://dx.doi.org/10.1007/BF02326825

[6] Nau, A., Scholtes, B., Evaluation of the High-Speed Drilling Technique for the Incremental HoleDrilling Method, Experimental Mechanics, 53, 531-542, 2013. http://dx.doi.org/10.1007/s11340012-9641-1

[7] Beghini, M., Bertini, L., Recent Advances in the Hole Drilling Method for Residual Stress Measurement, Journal of Materials Engineering and Performance, ASM International, 7, 163172, 1998. http://dx.doi.org/10.1361/105994998770347882

[8] Aoh, J.-N., Wei, C.-S., On the Improvement of Calibration Coefficients for Hole-Drilling Integral Method: Part I-Analysis of Calibration Coefficients Obtained by a 3-D FEM Model, Journal of Engineering Materials and Technology, ASME, Vol. 124, 250-258, 2002. http://dx.doi.org/10.1115/1.1416685 